\title{
High expression of PIG11 correlates with poor prognosis in gastric cancer
}

\author{
JUAN GU $^{1 *}$, SHU ZHANG ${ }^{2 *}$, XIN HE ${ }^{2},{\text { SUFANG } \mathrm{CHEN}^{3} \text { and YAN WANG }}^{2}$ \\ ${ }^{1}$ Department of Public Health, Jiangsu Vocational College of Medicine, Yancheng, Jiangsu 224005; \\ ${ }^{2}$ Department of Pathology, Affiliated Hospital of Nantong University, Nantong, Jiangsu 226001; \\ ${ }^{3}$ Department of Medical Imaging and Laboratory, Xiangnan University, Chenzhou, Hunan 423000, P.R. China
}

Received August 22, 2020; Accepted December 1, 2020

DOI: $10.3892 /$ etm.2021.9680

\begin{abstract}
P53-induced gene 11 (PIG11) is an early transcription-related target of p53 that is involved in cell apoptosis and tumor development. However, its biological function in gastric cancer (GC) tissues and relationship with the prognosis of patients with GC have remained elusive. In the present retrospective study, 60 fresh and 790 paraffin-embedded samples of GC were obtained from the Affiliated Hospital of Nantong University (Nantong, China) with complete clinical data from all patients. Reverse transcription-quantitative PCR and tissue microarray-immunohistochemical analysis were used to determine the expression of PIG11 in the respective GC tissues. A receiver operating characteristic (ROC) curve was plotted to determine the diagnostic utility of PIG11 expression in GC. Furthermore, three online databases, including Gene Expression Profiling Interactive Analysis (GEPIA), Oncomine and Kaplan-Meier plotter, were used for bioinformatics analysis of PIG11. PIG11 expression in GC tissues was high, which was positively correlated with invasive depth $(\mathrm{P}<0.001)$, lymph node metastasis $(\mathrm{P}<0.001)$, distant metastasis $(\mathrm{P}=0.019)$, TNM staging $(\mathrm{P}<0.001)$ and carcinoembryonic antigen in serum $(\mathrm{P}<0.001)$, and negatively associated with the overall survival of patients with GC. The ROC curve analysis suggested that based on PIG11 expression, it was possible to distinguish GC tissues from adjacent normal tissues $(\mathrm{P}<0.0001)$ with a sensitivity
\end{abstract}

Correspondence to: Dr Yan Wang, Department of Pathology, Affiliated Hospital of Nantong University, 20 Xisi Road, Nantong, Jiangsu 226001, P.R. China

E-mail: yisheng_wangyan@126.com

Dr Sufang Chen, Department of Medical Imaging and Laboratory, Xiangnan University, 889 Chenzhou Avenue, Chenzhou, Hunan 423000, P.R. China

E-mail: csf127@126.com

*Contributed equally

Key words: P53-induced gene 11, gastric cancer, tissue microarray, immunohistochemistry, prognosis and specificity of 81.67 and $76.67 \%$, respectively. In addition, Cox logistic regression analysis demonstrated that high PIG11 expression is a novel biomarker for unfavorable prognosis of patients with GC. Furthermore, the results obtained from the GEPIA database indicated that PIG11 expression is correlated with TNF, carcinoembryonic antigen related cell adhesion molecule 5, phosphatidylinositol-4, 5-bisphosphate 3-kinase catalytic subunit alpha, VEGFA and kinase insert domain receptor. Therefore, PIG11 expression may be associated with the malignancy of GC and may serve as a potential diagnostic and prognostic biomarker for GC.

\section{Introduction}

Gastric cancer (GC) is a common malignant tumor type of the digestive tract with high morbidity and mortality (1). The 5 -year survival probability of a patient with GC is $<20 \%$ (2). The risk factors for this cancer type are diverse, including genetics, gastroesophageal reflux disease, Helicobacter pylori infection, dietary factors and obesity (3). Although research on GC, particularly molecular research, has been increasing in recent years, the major methods of treatment are still conventional surgery, radiotherapy and chemotherapy (4). In addition, GC remains the fifth most frequently diagnosed neoplasm and the third most deadly tumor in the world (5). Due to in-depth research and rapid development of molecular biology to implement the use of novel treatments, including immunotherapy and targeted drugs, the current survival rate of patients with GC has improved (6), but these methods still have certain limitations, such as heterogeneity as well as poor sensitivity and specificity. Therefore, it is important to discover and optimize novel biomarkers for GC with high specificity and sensitivity.

The P53-induced gene 11 (PIG11), also known as tumor protein p53 induced protein 11 (TP53I11), is a target gene downstream of p53 located at human chromosome 11p11.2 (7,8). In 1997, Polyak et al (9) first identified that high expression of p53 significantly induced PIGs when studying the mechanism of p53-dependent apoptosis in human colon cancer cells using Serial Analysis of Gene Expression technology. The genes were named as PIG1-13 and PIG11 is one of them (9). In the PIG11 protein, a p53 DNA-binding site was detected, but no homologous proteins and conserved domains were evident, indicating that the protein may be novel $(10,11)$. 
In addition, the PIG11 gene, as a downstream target gene of p53, is closely related to p53-mediated apoptosis. In the human hepatic stellate cell line LX-1, the T antigen may cause p53 inactivation and reduce the expression of PIG11, which indicates that the expression of PIG11 protein is related to the p53 gene (12). Relevant studies suggested that the PIG11 protein is primarily distributed in the mammary gland, liver, gastrointestinal and other epithelial tissues and expressed in the corresponding cancer tissues $(13,14)$. Furthermore, PIG11 has a certain relationship with the occurrence and development of tumors, including non-small cell lung (15), breast (16) and liver cancer (14). However, the function of PIG11 in the process of GC and its impact on prognosis still require to be explored. In the present retrospective study reverse transcription-quantitative PCR (RT-qPCR) and tissue microarray-immunohistochemistry (TMA-IHC) were used to analyze PIG11 expression in GC tissues and determine the roles and clinical utility of PIG11 protein in patients with GC. Furthermore, online databases were used for bioinformatic analysis on PIG11 in GC.

\section{Materials and methods}

Patients. Freshly frozen GC tissues $(n=60)$ and matched adjacent normal tissues $(n=60)$ were obtained from the Department of Pathology of the Affiliated Hospital of Nantong University (Nantong, China). Clinicopathological data are presented in Table SI. They were randomly selected and from patients (41 males and 19 females; age range, 32-76 years; median age, 59.42 years) who had undergone GC resection at the Affiliated Hospital of Nantong University (Nantong, China) from January 2016 to June 2016. Furthermore, a total of 790 formalin-fixed ( $\sim 24 \mathrm{~h}$ after isolation) and paraffin-embedded samples from patients with GC (age range, 19-84 years; median age, 60.39 years) obtained between January 2004 and June 2009 stored at the Department of Pathology of the Affiliated Hospital of Nantong University (Nantong, China) were randomly collected. These samples included GC samples $(n=480)$ and non-cancerous samples (corresponding normal surgical margin samples selected from the 480 paraffin-embedded samples from patients with GC, $\mathrm{n}=60 ; 42$ males and 18 females, 35-82 years, median age, 63.15 years, high-grade intraepithelial neoplasia samples, $\mathrm{n}=72 ; 51$ males and 21 females, 41-81 years, median age, 60.76 years, low-grade intraepithelial neoplasia samples, $\mathrm{n}=56,46$ males and 10 females, 38-79 years, median age, 59.84 years; chronic gastritis samples, $\mathrm{n}=42,28$ males and 14 females, 29-74 years, median age, 52.48 years; intestinal metaplasia samples, $n=80,60$ males and 20 females, 25-82 years, median age, 56.43 years) that were used as control groups. Clinicopathological data are presented in Table SII. The cases included in the present study had a follow-up rate of $100 \%$ and clinicopathological information, including sex, age, body mass index (BMI), histological type, differentiation, invasive depth (T-stage), lymph node metastasis (N-stage), distant metastasis (M-stage), TNM stage, carcinoembryonic antigen (CEA), carbohydrate antigen 19-9 (CA19-9), human epidermal growth factor receptor 2 (HER-2) and Laurén classification. All diagnoses were confirmed by two independent pathologists at the Department of Pathology of the Affiliated
Hospital of Nantong University (Nantong, China) according to the most recent World Health Organization classification criteria (17) and the 8th edition of the TNM Classification of Malignant Tumors (18). Overall survival (OS) was assessed, with survival defined as the duration from the time of surgical resection to death or the end of the follow-up period. None of the patients enrolled received any radiation, chemotherapy or immunotherapy prior to surgery. A written informed consent form was signed by each patient prior to the use of their sample for scientific research. The present study was approved by the Human Research Ethics Committee at the Affiliated Hospital of Nantong University (Nantong, China). All experimental methods and related protocols were performed according to the regulations of the Affiliated Hospital of Nantong University (Nantong, China).

Gene expression profiling interactive analysis (GEPIA) and oncomine database online analysis. GEPIA and Oncomine were used to analyze the expression of PIG11 mRNA in GC. GEPIA (http://gepia.cancer-pku.cn/) is able to analyze the RNA sequencing expression data of 9,736 tumors and 8,587 normal samples with a standard processing pipeline (19), in addition to providing differential expression models for tumors and normal tissues. In this database, the expression of PIG11 in GC was analyzed. The terms 'PIG11', 'mRNA', 'GC' and 'Cancer vs. Normal Analysis' were selected to query the Oncomine database (http://www.oncomine.org) and obtain the mRNA expression data for PIG11 in GC. The Oncomine database is a large tumor gene chip database, covering 65 gene chip data sets, 4,700 chips and 480 million gene expression data. In this database, gene expression differences may be analyzed, co-expressed genes may be predicted and possible molecular markers and therapeutic targets may be determined in analyses based on known gene-drug interactions.

$R T$ - $q P C R$ analysis. Total RNA from the 60 pairs of fresh GC tissues and matched adjacent normal tissues was extracted with TRIzol (cat. no. 79306; Gibco; Thermo Fisher Scientific, Inc.). cDNA was synthesized using commercial kits (PrimeScript RT Reagent Kit with gDNA Eraser; cat. no. RR047A; Takara Biotechnology Co., Ltd.) according to the manufacturer's protocol. RT-qPCR analysis was performed using an ABI 7500 system (Applied Biosystems; Thermo Fisher Scientific, Inc.) with the SYBRR ${ }^{\circledR}$ Premix ExTaqkit (Takara Bio, Inc.) in 96-well plates. The total reaction volume was $20 \mu \mathrm{l}$. The specific primer sequences for PIG11 were as follows: Upstream primer, 5'-GCGAATTCCAACACCGATGCACACA-3' and downstream, 5'-CGCGGATCCTAGGCAGCTCTTTAGG-3'. $\beta$-actin was used as an internal control in this analysis, for which the upstream primer was 5'-TAATCTTCGCCTTAA TACTT-3' and the downstream primer was 5'-AGCCTTCAT ACATCTCAA-3'. The reaction conditions were as follows: $10 \mathrm{~min}$ at $95^{\circ} \mathrm{C}$, followed by 40 cycles of $95^{\circ} \mathrm{C}$ for $10 \mathrm{sec}$ and $45 \mathrm{sec}$ at $60^{\circ} \mathrm{C}$. Relative expression levels were calculated using the $2^{-\triangle \Delta C q}$ method (20). All experiments were repeated in triplicate.

TMA-IHC analysis. TMAs were constructed as previously described (21). Samples (2 $\mathrm{mm}$ in diameter) were sliced into $4-\mu \mathrm{m}$ sections for IHC analysis. The sections were 


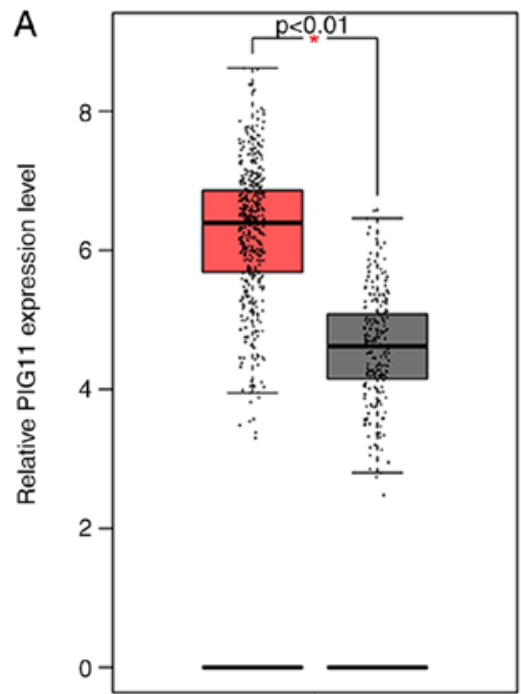

B

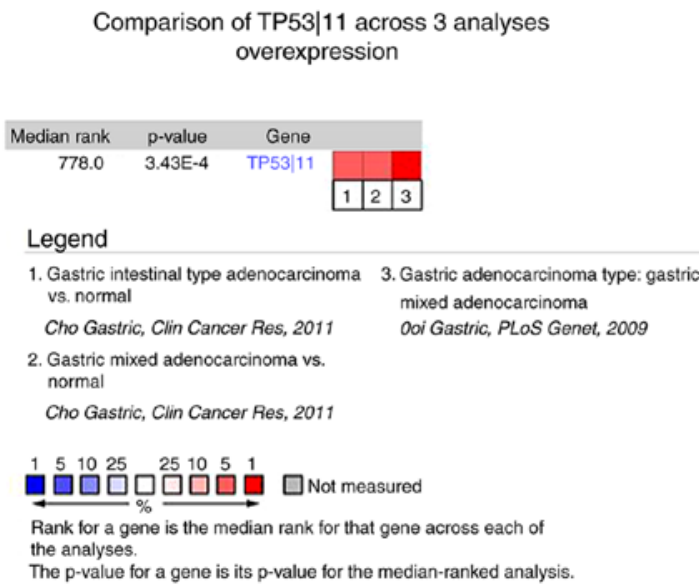

Figure 1. PIG11 mRNA expression in GC and normal tissues obtained from online databases. (A) PIG11 mRNA expression obtained from the Gene Expression Profiling Interactive Analysis database in GC tissues (red, $\mathrm{n}=408$ ) and normal tissues (black, $\mathrm{n}=211$ ). (B) PIG11 mRNA analyzed by Cho et al (25) (gastric adenocarcinoma, $\mathrm{n}=65$; surrounding normal tissues, $\mathrm{n}=19$; gastrointestinal stromal tumor samples, $\mathrm{n}=6$ ) and Ooi et al (26) (GCs, $\mathrm{n}=31)$ provided by the Oncomine database. PIG11/TP53I11, P53-induced gene 11/tumor protein p53 induced protein 11; GC, gastric cancer.

deparaffinized with xylene and rehydrated in an ethanol gradient, followed by washing with PBS (0.01 M, pH 7.0), boiling under pressure in citrate buffer $(0.01 \mathrm{M}, \mathrm{pH}$ 6.0) for antigen retrieval and incubation in PBS with 5\% goat serum (cat. no. SL039; Beijing Solarbio Science \& Technology Co., Ltd.) for $30 \mathrm{~min}$ at $37^{\circ} \mathrm{C}$ to block non-specific binding. Next, the sections were stained with polyclonal rabbit anti-PIG11 antibody (dilution, 1:200; cat. no. PA5-51336; Invitrogen; Thermo Fisher Scientific, Inc.) at $4^{\circ} \mathrm{C}$ overnight and then with HRP-labeled goat anti-rabbit IgG (1:2,500; cat. no. ZDR-5306; OriGene Technologies, Inc.) secondary antibody for $30 \mathrm{~min}$ at room temperature. The sections were subsequently incubated with diaminobenzidine (grant no. DA1010; Beijing Solarbio Science \& Technology Co., Ltd.) for $8 \mathrm{~min}$ at room temperature and finally counterstained with hematoxylin. A total of two pathologists (SZ and YW) under blinded experimental conditions assessed the expression of PIG11 across the entire surface of the slide on every chip. The rating standard for the staining intensity was as follows: 0 , negative; 1 , weakly positive; 2 , medium positive; and 3 , strongly positive. The positive rate score was assigned as follows: 0 , lowest; to 100 , highest. The final score was the product of the two above-mentioned scores, which ranged from 0 to 300 . X-tile (Rimm laboratory at Yale University; http://www.tissuearray.org/rimmlab) (22) software, a new bioinformatics tool for biomarker assessment and outcome-based cut-point optimization, was used to set the final cutoff value for the PIG11 expression data. The cutoff value was determined based on the maximum $\chi^{2}$ value and estimated by log-rank $\chi^{2}$ statistics according to OS. Finally, 140 was selected as the cut-off point to stratify samples: $0-140$, low or no expression; and 141-300, high expression.

Bioinformatics analysis. Kaplan-Meier plotter and GEPIA were used for bioinformatics analysis of PIG11 in GC. Kaplan-Meier plotter (http://kmplot.com/analysis/) (23) was utilized to assess the prognostic role of PIG11 expression in patients with GC. The Kaplan-Meier plotter is able to assess the effect of 54,000 genes (mRNAs, miRNAs, proteins) on survival in 21 cancer types, including breast $(\mathrm{n}=6,234)$, ovarian $(n=2,190)$ and lung cancer $(n=3,452)$, as well as GC $(n=1,440)$, which is used for meta-analysis-based discovery and validation of survival biomarkers. In the GEPIA database, the relationships between PIG11 expression in tumors and the OS of patients were analyzed. In addition, the correlations of PIG11/TP53I11 with TNF, CEA-related cell adhesion molecule 5 (CEACAM5), phosphatidylinositol-4,5-bisphosphate 3-kinase catalytic subunit alpha (PIK3CA), VEGFA and kinase insert domain receptor (KDR; also known as VEGF receptor-2) which may be related to the development of GC and molecular typing (24) in GC were further assessed in this database. 'Pearson' was selected for 'Correlation Coefficient' and 'Stomach adenocarcinoma (STAD) Tumor' was selected as 'Used Expression Datasets'.

Statistical analysis. SPSS 22.0 (IBM Corp.) and GraphPad 5.0 (GraphPad Software, Inc.) were used for statistical analysis. Wilcoxon's signed-rank non-parametric test was utilized to analyze the results obtained by RT-qPCR. The diagnostic value of PIG11 expression in GC was estimated using receiver operating characteristic (ROC) curve analysis. MedCalc software was used for ROC curve analysis. The $\chi^{2}$ test was used to determine the relationship between PIG11 expression and clinicopathological parameters. The Kaplan-Meier method and the log-rank test were utilized to generate survival curves. The factors of prognostic significance in the univariate analysis were further assessed using the multivariate Cox regression model. For all of the above-mentioned tests, $\mathrm{P}<0.05$ was considered to indicate statistical significance.

\section{Results}

Expression of PIGIImRNA in GC tissues from online databases. The results obtained from GEPIA (GC tissues, $n=408$; normal tissues, $n=211$ ), together with analyses of 

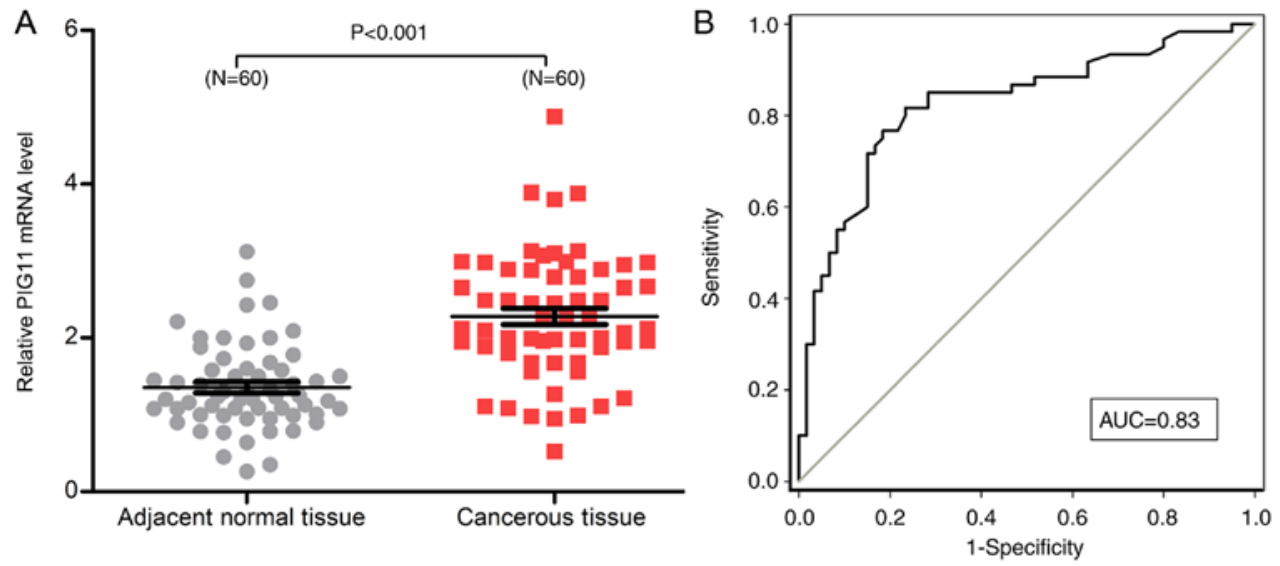

Figure 2. Expression pattern and diagnostic value of PIG11 in fresh GC tissues assessed by reverse transcription-quantitative PCR in the present study. (A) PIG11 mRNA was significantly higher in GC tissues $(n=60,2.28 \pm 0.82)$ than that in adjacent normal tissues $(n=60,1.36 \pm 0.55$; $P<0.05)$. (B) ROC curve analysis demonstrated that PIG11 was able to distinguish GC tissues from adjacent normal tissues. The cut-off value for the AUC was 1.635. ROC, receiver operating characteristic; AUC, area under the ROC curve; PIG11, P53-induced gene 11; GC, gastric cancer.

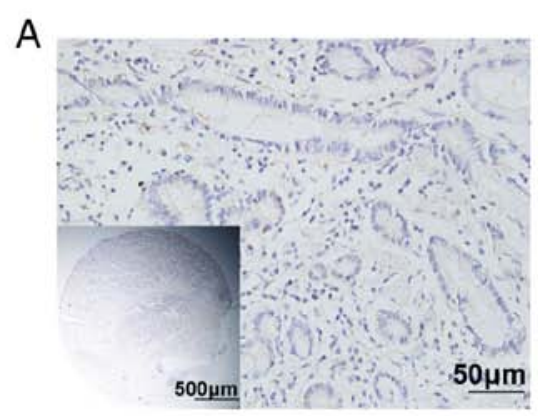

$\mathrm{D}$

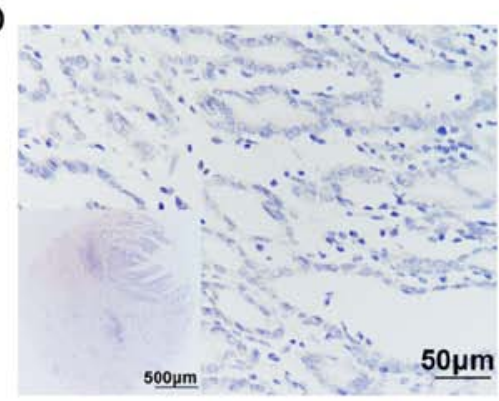

B

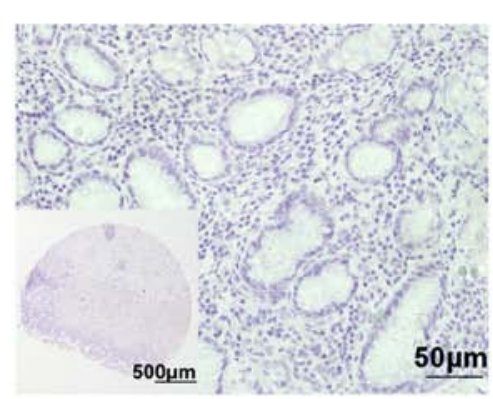

E

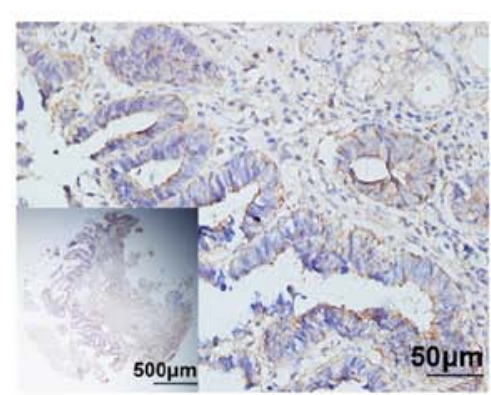

C

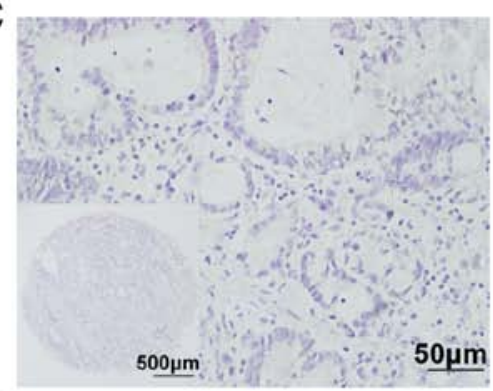

F

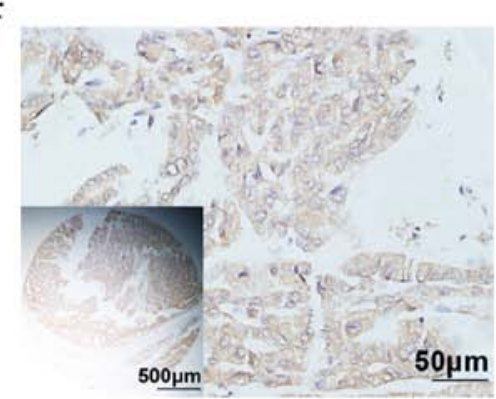

Figure 3. Immunohistochemical images for the detection of PIG11 protein expression in GC and non-cancerous tissues in the present study. (A) In chronic gastritis tissues $(n=42),(B)$ intestinal metaplasia tissues $(n=80),(C)$ surgical margin tissues $(n=60)$ and (D) low-grade intraepithelial neoplasia ( $n=56)$, weak expression was observed. (E) In high-grade intraepithelial neoplasia $(n=72)$ and $(\mathrm{F}) \mathrm{GC}$ tissues $(\mathrm{n}=480)$, positive expression was observed. PIG11 protein was high in cancerous tissues. Original magnifications of x400 (scale bar, $50 \mu \mathrm{m}$ ) and x40 (scale bar, 500 $\mu \mathrm{m}$ ). PIG11, P53-induced gene 11; GC, gastric cancer.

data from Cho et al (25) (gastric adenocarcinoma, $\mathrm{n}=65$; surrounding normal tissues, $\mathrm{n}=19$; gastrointestinal stromal tumor samples, $\mathrm{n}=6)$ and Ooi et al $(26)(\mathrm{GCs}, \mathrm{n}=31)$ provided by Oncomine both indicated that PIG11 mRNA expression in cancerous tissues was higher than that in normal tissues (both $\mathrm{P}<0.05$; Fig. $1 \mathrm{~A}$ and $\mathrm{B})$.

Expression and diagnostic value of PIG11mRNA in GC. RT-qPCR analysis of the freshly frozen tissues from the 60 patients with GC indicated that the relative mRNA expression of PIG11 in cancerous tissues was higher $(2.28 \pm 0.82)$ than that in adjacent normal tissues $(1.36 \pm 0.55$; $\mathrm{P}<0.05$; Fig. 2A). ROC curve analysis was adopted to estimate the diagnostic value of PIG11 in GC. As presented in Fig. 2B, PIG11 expression was able to distinguish GC tissues from adjacent normal tissues, with an area under the curve of 0.83 (95\% CI, 0.7504-0.9023) at a cut-off value of 1.635. The corresponding sensitivity and specificity were 81.67 and $76.67 \%$, respectively.

PIG11 protein expression in GC and correlation with the clinical characteristics of GC. According to the TMA-IHC analysis (representative images provide in Fig. 3), the percentage of samples with PIG11 expression observed in the cytoplasm was greater among GC tissues (340/480, 70.8\%) compared with that among chronic gastritis (11/42, 26.2\%), intestinal metaplasia $(22 / 80,27.5 \%)$, low-grade intraepithelial neoplasia $(18 / 56,32.1 \%)$, high-grade intraepithelial neoplasia 
Table I. Expression of P53-induced gene 11 determined by immunohistochemistry in GC and gastric non-cancerous tissues a cohort of the present study $(n=790)$.

\begin{tabular}{lcccc}
\hline Tissue type & $\mathrm{N}$ & Low or no expression & High expression & Pearson $\chi^{2}$ \\
\hline Chronic gastritis & 42 & $31(73.8)$ & $11(26.2)$ & 119.363 \\
Intestinal metaplasia & 80 & $58(72.5)$ & $22(27.5)$ & $<0.001$ \\
Low-grade intraepithelial neoplasia & 56 & $38(67.9)$ & $18(32.1)$ & $36(50.0)$ \\
High-grade intraepithelial neoplasia & 72 & $36(50.0)$ & $340(70.8)$ \\
GC & 480 & $140(29.2)$ & $16(26.7)$ \\
Surgical margin (GC patients) & 60 & $44(73.3)$ & \\
\hline
\end{tabular}

Values are expressed as n (\%). GC, gastric cancer.
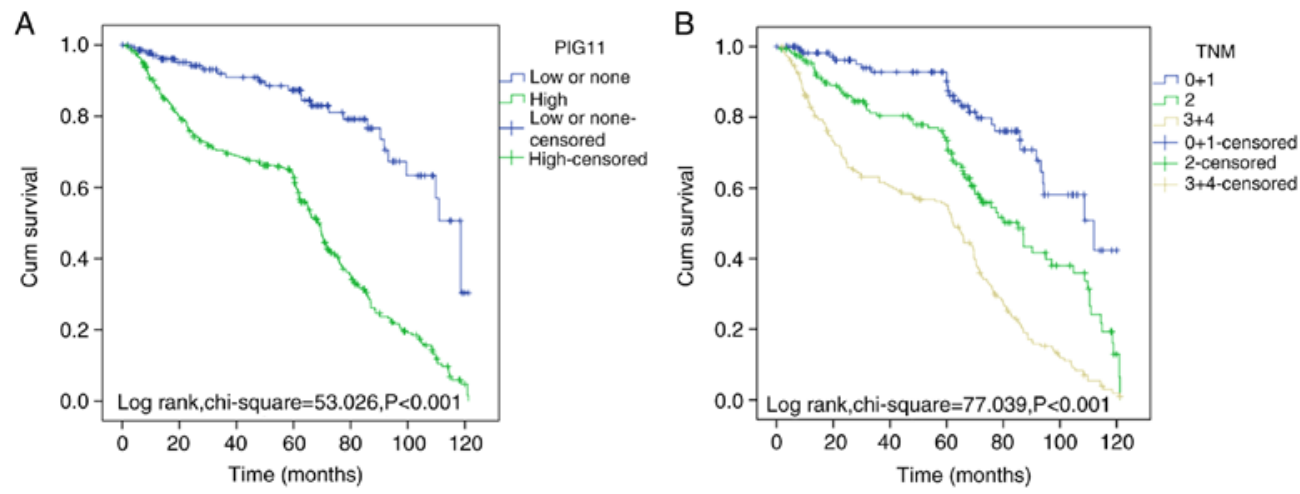

Figure 4. Survival analysis of patients with GC from the present cohort (patients with GC subjected to immunohistochemical analysis). (A) Patients with GC and high PIG11 expression (green line, $n=340$ ) had worse overall survival than the patients with low PIG11 expression (blue line, $n=140$ ). (B) Patients with advanced TNM stage had a relatively shorter survival time $(0+1, n=123 ; 2, n=154 ; 3+4, n=203)$. Censored datapoints indicate patients who were still alive until the end of follow-up. PIG11, P53-induced gene 11; GC, gastric cancer; Cum, cumulative.

$(36 / 72,50 \%)$ and surgical margin tissues $(16 / 60,26.7 \%)$, indicating that PIG11 expression in GC was elevated(Pearson $\chi^{2}=119.363, \mathrm{P}<0.001$; Table I). As presented in Table II, PIG11 expression was positively correlated with infiltration depth (T-stage, $\mathrm{P}<0.001)$, lymph node involvement $(\mathrm{N}$-stage, $\mathrm{P}<0.001)$, metastasis (M-stage, $\mathrm{P}=0.019)$, TNM stage $(\mathrm{P}<0.001)$ and CEA in bloodserum $(\mathrm{P}<0.001)$, but not with sex, age, BMI, histological type, tumor differentiation, CA19-9 levels in blood serum, HER-2 and Laurén classification.

Association between high PIG11 expression and prognosis of patients with $G C$. The results of the univariate analyses suggested that high PIG11 expression [hazard ratio $(\mathrm{HR})=3.919$; $\mathrm{P}<0.001]$, tumor differentiation $(\mathrm{HR}=1.421 ; \mathrm{P}<0.001)$, T-stage $(\mathrm{HR}=1.675 ; \mathrm{P}<0.001), \mathrm{N}$-stage $(\mathrm{HR}=1.470 ; \mathrm{P}<0.001), \mathrm{M}$-stage $(\mathrm{HR}=1.944 ; \mathrm{P}=0.007)$ and TNM stage $(\mathrm{HR}=2.115 ; \mathrm{P}<0.001)$ were associated with poor OS of patients with GC (Table III). Further multivariate analysis indicated that high PIG11 expression $(\mathrm{HR}=2.926 ; \mathrm{P}<0.001)$ and $\mathrm{TNM}$ stage $(\mathrm{HR}=1.578$; $\mathrm{P}=0.021)$ were independent prognostic factors for $\mathrm{GC}$ (Table III).

Kaplan-Meier curve analysis for the present cohort (patients with GC whose samples were subjected to IHC analysis, cutoff value $=140$ ) suggested that high PIG11 expression and advanced TNM stage were associated with poor prognosis of GC (both $\mathrm{P}<0.001$; Fig. 4). Furthermore, the results obtained from the Kaplan-Meier plotter database $(n=875)$ indicated that high PIG11 expression was associated with poor prognosis of GC $(\mathrm{P}<0.001$; Fig. 5$)$, using the median as the cutoff value to divide patients into a low- and a high-expression group.

Survival analysis for PIG11 in different tumor types. Based on the data analyzed on GEPIA, high PIG11 expression was associated with an unfavorable survival prognosis of patients with breast invasive carcinoma $[\mathrm{n}=1,070, \log$-rank $\mathrm{P}=0.00017, \mathrm{HR}$ (high) $=1.90]$, kidney renal papillary cell carcinoma $[\mathrm{n}=282, \log$ rank $\mathrm{P}=0.031$, HR (high) $=2.00]$, mesothelioma $[\mathrm{n}=82$, log-rank $\mathrm{P}=0.04$, HR (high) $=1.70]$, uveal melanoma $[\mathrm{n}=78$, log-rank $\mathrm{P}=0.014$, HR (high)=3.10], adopting the 'median' as the cutoff value to divide patients into a low- and a high-expression group (Fig. 6). Correlation analysis of PIG11 with TNF, CEACAM5, PIK3CA, VEGFA and KDR demonstrated that in GC tissues $(n=408)$, PIG11 expression was significantly correlated with TNF $(\mathrm{P}=0.0011, \mathrm{R}=0.16)$, CEACAM5 $\left(\mathrm{P}=7.9 \times 10^{-5}, \mathrm{R}=0.19\right)$, PIK3CA $(\mathrm{P}=0.028, \mathrm{R}=0.11)$, VEGFA $(\mathrm{P}=0.004, \mathrm{R}=0.14)$ and $\mathrm{KDR}\left(\mathrm{P}=1.7 \times 10^{-5}, \mathrm{R}=0.21\right)$, as presented in Fig. 7 .

\section{Discussion}

GC is a common upper gastrointestinal tumor type (27). Given the atypicality of clinical symptoms, numerous patients are already in the stage of advanced metastasis at the time of clear 
Table II. Association of P53-induced gene 11 expression determined by tissue microarray-immunohistochemistry with clinical characteristics in patients with gastric cancer in a cohort of the present study.

\begin{tabular}{|c|c|c|c|c|c|}
\hline Characteristics & $\mathrm{n}$ & Low or no expression & High expression & Pearson $\chi^{2}$ & P-value \\
\hline Total & 480 & $140(29.2)$ & $340(70.8)$ & & \\
\hline Sex & & & & 0.454 & 0.501 \\
\hline Male & 353 & $100(28.3)$ & $253(71.7)$ & & \\
\hline Female & 127 & $40(31.5)$ & $87(68.5)$ & & \\
\hline Age (years) & & & & 0.819 & 0.366 \\
\hline$\leq 60$ & 228 & $71(31.1)$ & $157(68.9)$ & & \\
\hline$>60$ & 252 & $69(27.4)$ & $183(72.6)$ & & \\
\hline $\operatorname{BMI}\left(\mathrm{kg} / \mathrm{m}^{2}\right)$ & & & & 0.063 & 0.082 \\
\hline$\leq 24$ & 181 & $54(29.8)$ & $127(70.2)$ & & \\
\hline$>24$ & 299 & $86(28.8)$ & $213(71.2)$ & & \\
\hline Histological type & & & & 5.131 & 0.162 \\
\hline Tubular & 409 & $125(30.6)$ & $284(69.4)$ & & \\
\hline Mucinous & 21 & $2(9.5)$ & $19(90.5)$ & & \\
\hline Mixed (tubular and mucinous) & 20 & $4(20.0)$ & $16(80.0)$ & & \\
\hline Signet ring cell & 30 & $9(30.0)$ & $21(70.0)$ & & \\
\hline Differentiation & & & & 4.471 & 0.107 \\
\hline Well & 62 & $22(35.5)$ & $40(64.5)$ & & \\
\hline Intermediate & 281 & $87(31.0)$ & $194(69.0)$ & & \\
\hline Poor & 137 & $31(22.6)$ & $106(77.4)$ & & \\
\hline T-stage & & & & 71.189 & $<0.001$ \\
\hline 1 (Tis+T1) & 86 & $55(64.0)$ & $31(36.0)$ & & \\
\hline 2 & 86 & $28(32.6)$ & $58(67.4)$ & & \\
\hline 3 & 264 & $54(20.5)$ & $210(79.5)$ & & \\
\hline 4 & 44 & $3(6.8)$ & $41(93.2)$ & & \\
\hline $\mathrm{N}$-stage & & & & 31.838 & $<0.001$ \\
\hline 0 & 201 & $84(41.8)$ & $117(58.2)$ & & \\
\hline 1 & 70 & $21(30.0)$ & $49(70.0)$ & & \\
\hline 2 & 115 & $22(19.1)$ & $93(80.9)$ & & \\
\hline 3 & 94 & $13(13.8)$ & $81(86.2)$ & & \\
\hline M-stage & & & & 5.471 & 0.019 \\
\hline 0 & 461 & $139(30.2)$ & $322(69.8)$ & & \\
\hline 1 & 19 & $1(5.3)$ & $18(94.7)$ & & \\
\hline TNM stage & & & & 65.348 & $<0.001$ \\
\hline $0+1$ & 123 & $68(55.3)$ & $55(44.7)$ & & \\
\hline 2 & 154 & 45 (29.2) & 109 (70.8) & & \\
\hline $3+4$ & 203 & $27(13.3)$ & $176(86.7)$ & & \\
\hline CEA (ng/ml) & & & & 21.404 & $<0.001$ \\
\hline$\leq 5$ & 241 & $83(34.4)$ & 158 (65.6) & & \\
\hline$>5$ & 198 & 37 (18.7) & $161(81.3)$ & & \\
\hline Unknown & 41 & $20(48.8)$ & $21(51.2)$ & & \\
\hline CA19-9 (U/ml) & & & & 1.179 & 0.555 \\
\hline$\leq 37$ & 197 & $62(31.5)$ & $135(68.5)$ & & \\
\hline$>37$ & 245 & $69(28.2)$ & $176(71.8)$ & & \\
\hline Unknown & 38 & $9(23.7)$ & $29(76.3)$ & & \\
\hline HER-2 & & & & 0.008 & 0.931 \\
\hline $0-2$ & 388 & $113(29.1)$ & 275 (70.9) & & \\
\hline 3 & 92 & $27(29.3)$ & 65 (70.7) & & \\
\hline
\end{tabular}


Table II. Continued.

\begin{tabular}{lrccc}
\hline Characteristics & $\mathrm{n}$ & Low or no expression & High expression & Pearson $\chi^{2}$ \\
\hline Laurén classification & & & & 4.465 \\
$\quad$ Intestinal type & 263 & $87(33.1)$ & $176(66.9)$ & 0.107 \\
Mixed type & 83 & $19(22.9)$ & $64(77.1)$ & \\
Diffuse type & 134 & $34(25.4)$ & $100(74.6)$ & \\
\hline
\end{tabular}

Values are expressed as n (\%). HER-2, human epidermal growth factor receptor 2; BMI, body mass index.

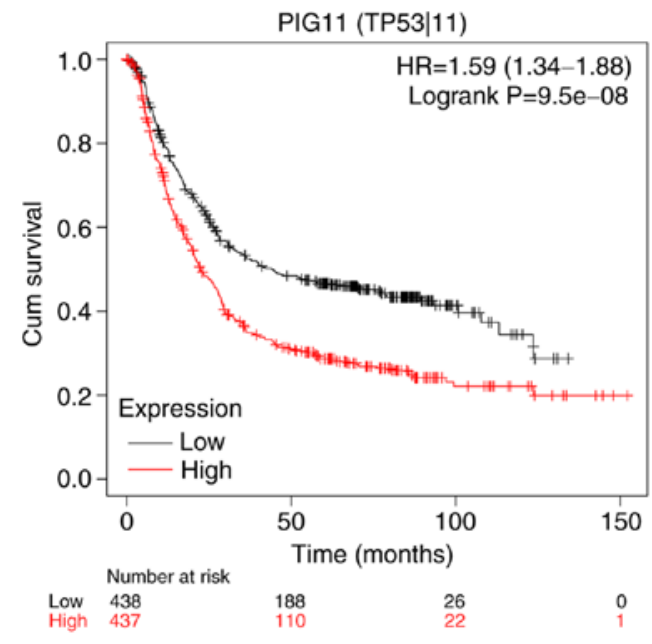

Figure 5. Survival analysis of patients with GC using the Kaplan-Meier plotter online database. The overall survival of patients with GC and high PIG11 expression (red line, $n=437$ ) was significantly lower than that of patients with GC and low PIG11 expression (black line, $\mathrm{n}=438$ ). Censored datapoints indicate patients who were still alive until the end of follow-up, patients who did not show up to follow up appointments and patients who died of other causes before the end of follow-up. PIG11, P53-induced gene 11; GC, gastric cancer; HR, hazard ratio (provided with 95\% CI); Cum, cumulative.

diagnosis and metastatic GC is closely related to poor prognosis (28). An effective treatment plan for GC is necessary. At present, with the emergence of various biomarkers for $\mathrm{GC}$, such as VEGFR2 (29), VEGF (30,31), EGFR (32), MET (33), PIK3CA (34) and mTOR (35), the corresponding targeted drugs have brought new hope regarding the OS of patients. Successful biomarkers require to be cost-effective and reproducible, but to date, challenges in identifying suitable predictive biomarkers have hampered the success of numerous targeted therapies for GC (28). Therefore, it is necessary to discover novel biomarkers that may accurately predict patient outcomes and allow for better treatment and follow-up decisions.

PIG11 is a downstream target gene in the p53-mediated apoptotic pathway. The expression of PIG11 is closely related to the high expression of p53 in colon cancer and cell apoptosis is involved (9). The PIG11 gene is located on the human chromosome 11p11.2 (7) and the encoded protein is located in the cytoplasm, which contains 121 amino acids. Its isoelectric point is PI 8.24 and its molecular weight is $12.904 \mathrm{kDa}$. As indicated in a PATCH software analysis, PIG11 exists in the DNA-binding sites of the gene p53, while a Basic Local Alignment Search Tool (https://blast.ncbi.nlm.nih.gov/Blast. cgi) search indicated that there are no records for PIG11 protein in this database. It has been illustrated in studies that PIG11 is mainly expressed in normal and tumor epithelial cells and may be involved in the occurrence and development of tumors because of its role in apoptosis (15).

GC is divided into four molecular subgroups, namely Epstein-Barr virus-positive, microsatellite instability, chromosomal instability (CIN) and genomic stable, according to The Cancer Genome Atlas (TCGA) (36). At present, the latter two subgroups cannot be identified in the presence or absence of extensive somatic copy number changes in TCGA (37). CIN, as the driving force of intratumoral heterogeneity, is closely related to cancer drug resistance (38). Therefore, GC with CIN must be identified. According to TCGA, TP53 mutation and the loss of its protein pathway are among the key features of CIN GC (37). PIG11, which is regarded as a TP53-induced protein, may be an important diagnostic tool for this group.

P53 is associated with high proliferation of tumor cells (39). Cell apoptosis induced by p53 is carried out in a complex multi-step process. The activation of p53 induced by redox-related genes, production of reactive oxygen species and mitochondrial oxidative damage are involved in the process $(40,41)$. Studies have indicated that numerous proteins encoded by PIGs are able to generateor regulate oxidative stress. PIG1 encodes galactin-7 and stimulates the production of superoxide (42). PIG6 is involved in the prolongation of oxidative stress (43). PIG8 induced by p53 is considered to be a tumor-suppressor gene (44). All of the above-mentioned PIG species are closely related to the intracellular redox status, thereby indicating that PIG11 may be related to oxidative reduction and mitochondrial component damage during apoptosis. As reported in another study, overexpression of PIG11 may change metabolic pathways and metabolites of redox processes, such as the changes of NADPH oxidase in the membrane (45).

Apoptosis of epithelial cells depends on the attachment of integrin-mediated extracellular matrix (ECM) (46). Its neoplastic transformation is associated with loss of neurodegeneration and increased ECM-dependent survival, which is widely regarded as a sign of oncogenic epithelial-mesenchymal transition (EMT) necessary for epithelial cancer stem cells and tumor metastasis (47). Cell death induced by ECM deprivation involves intrinsic and extrinsic apoptosis and autophagy (48). Furthermore, PIG11 is related to EMT, cell migration and invasion in MCF10A cells (49).

In the present study, PIG11 expression in GC tissues was significantly higher than that in the non-cancerous tissues. 
Table III. Univariate and multivariate analysis of prognostic factors influencing 5-year survival of patients with gastric cancer who donated IHC samples in a cohort of the present study.

\begin{tabular}{|c|c|c|c|c|c|c|}
\hline \multirow[b]{2}{*}{ Factor } & \multicolumn{3}{|c|}{ Univariate analysis } & \multicolumn{3}{|c|}{ Multivariate analysis } \\
\hline & HR & P-value & $95 \% \mathrm{CI}$ & HR & P-value & $95 \% \mathrm{CI}$ \\
\hline PIG11 expression (high vs. low and none) & 3.919 & $<0.001$ & $2.633-5.833$ & 2.926 & $<0.001$ & $1.941-4.410$ \\
\hline Age ( $\leq 60$ vs. $>60$ years $)$ & 1.193 & 0.150 & $0.938-1.516$ & & & \\
\hline Sex (male vs. female) & 1.104 & 0.472 & $0.843-1.446$ & & & \\
\hline BMI ( $\leq 24$ vs. $\left.>24 \mathrm{~kg} / \mathrm{m}^{2}\right)$ & 1.213 & 0.133 & $0.943-1.560$ & & & \\
\hline $\begin{array}{l}\text { Histological type [tubular vs. mucinous vs. mixed } \\
\text { (tubular and mucinous) vs. signet ring cells] }\end{array}$ & 1.038 & 0.621 & $0.894-1.206$ & & & \\
\hline Differentiation (well vs. intermediate vs. poor) & 1.421 & $<0.001$ & $1.178-1.714$ & 1.065 & 0.680 & $0.791-1.434$ \\
\hline TNM stage $(0+1$ vs. 2 vs. $3+4)$ & 2.115 & $<0.001$ & $1.771-2.526$ & 1.578 & 0.021 & $1.071-2.327$ \\
\hline T-stage ( 1 vs. 2 vs. 3 vs. 4 ) & 1.675 & $<0.001$ & $1.433-1.956$ & 1.014 & 0.913 & $0.794-1.294$ \\
\hline $\mathrm{N}$-stage (0 vs. 1 vs. 2 vs. 3 ) & 1.470 & $<0.001$ & $1.329-1.626$ & 1.121 & 0.197 & $0.942-1.335$ \\
\hline M-stage (0 vs. 1$)$ & 1.944 & 0.007 & $1.204-3.139$ & 1.163 & 0.559 & $0.701-1.926$ \\
\hline CEA level (ng/ml) ( $\leq 5$ vs. $>5$ ) & 0.962 & 0.686 & $0.800-1.159$ & & & \\
\hline CA19-9 level (U/ml) ( $\leq 37$ vs. $>37)$ & 1.051 & 0.603 & $0.872-1.266$ & & & \\
\hline HER-2 level (0-2 vs. 3) & 1.081 & 0.612 & $0.800-1.461$ & & & \\
\hline $\begin{array}{l}\text { Laurén classification (intestinal type vs. mixed } \\
\text { type vs. diffuse type) }\end{array}$ & 1.275 & $<0.001$ & $1.112-1.462$ & 1.179 & 0.155 & $0.940-1.479$ \\
\hline
\end{tabular}

PIG11, P53-induced gene 11; HER-2, human epidermal growth factor receptor 2; BMI, body mass index.
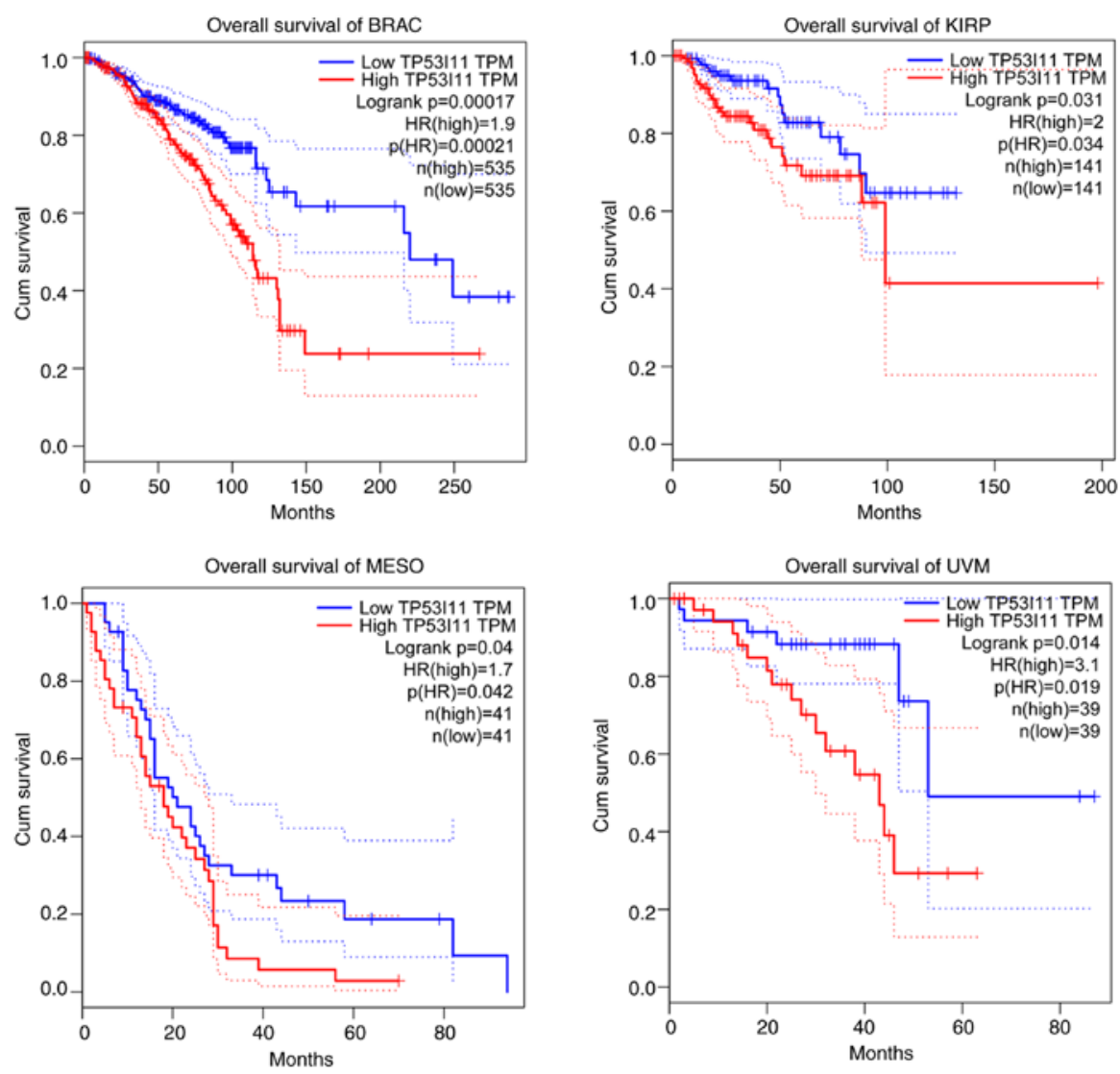

Figure 6. Association between the expression of PIG11 and prognosis of patients with different tumor types obtained from the Gene Expression Profiling Interactive Analysis online database. The overall survival of patients with BRAC ( $\mathrm{n}=1,070), \operatorname{KIRP}(\mathrm{n}=282), \operatorname{MESO}(\mathrm{n}=82)$ and UVM ( $\mathrm{n}=78)$ with high PIG11 expression (red line) was significantly lower than that of patients with low PIG11 expression (blue line). Censored datapoints indicate patients who were still alive until the end of follow-up, patients who did not show up to follow up appointments and patients who died of other causes before the end of follow-up. Dotted lines indicate the 95\% CI. BRAC, breast invasive carcinoma; KIRP, kidney renal papillary cell carcinoma; MESO, mesothelioma; UVM, uveal melanoma; PIG11, P53-induced gene 11; HR, hazard ratio; Cum, cumulative. 

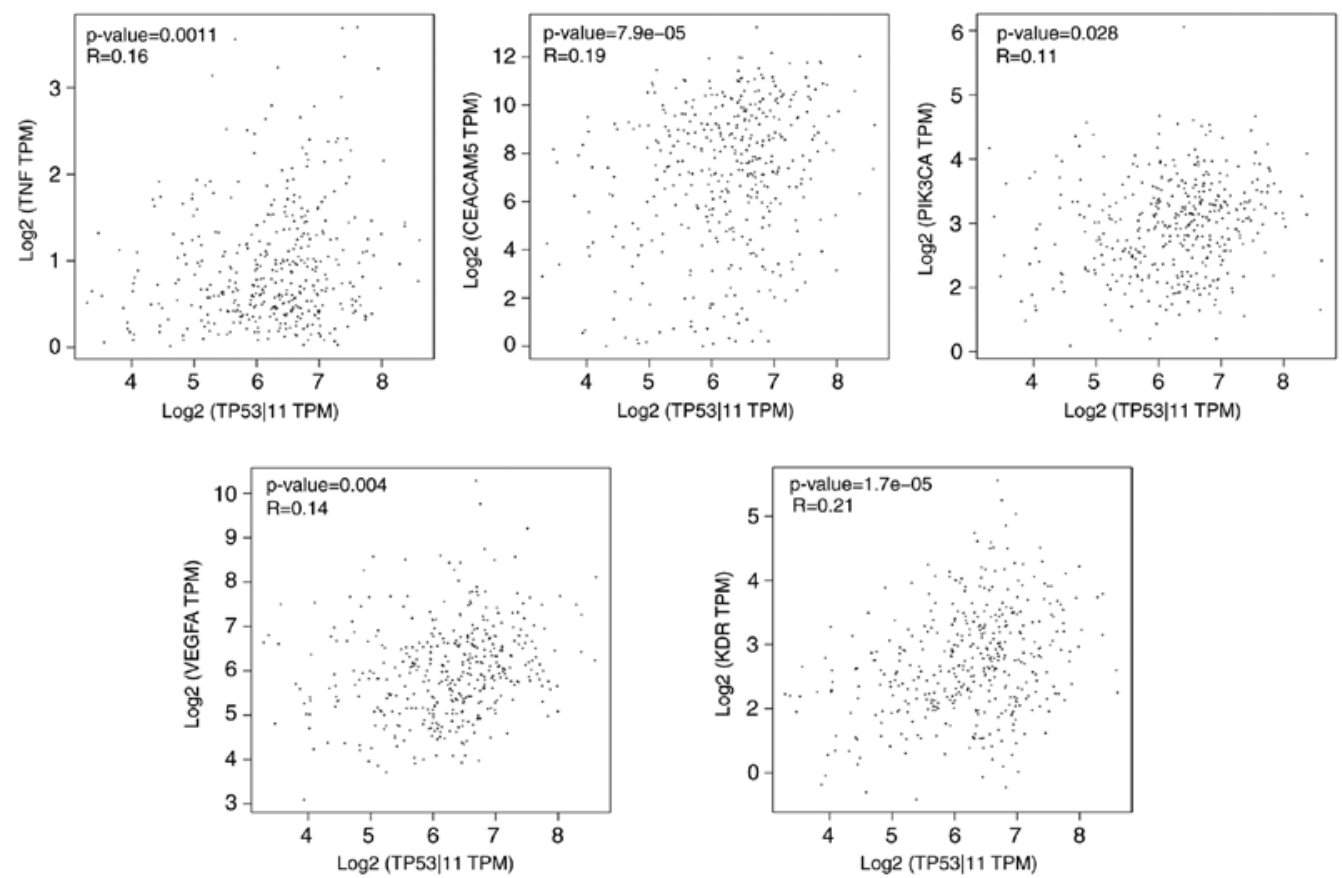

Figure 7. Correlationsamong PIG11, TNF, CEACAM5, PIK3CA, VEGFA and KDR in GC tissues obtained from the Gene Expression Profiling Interactive Analysis online database. In GC tissues $(\mathrm{n}=408)$, the expression of PIG11 was significantly correlated with that of TNF, CEACAM5, PIK3CA, VEGFA and KDR. TPM, transcripts per kilo base of the exon model per million mapped reads; PIG11/TP53I11, P53-induced gene 11; CEACAM5, carcinoembryonic antigen related cell adhesion molecule 5; PIK3CA, phosphatidylinositol-4,5-bisphosphate 3-kinase catalytic subunit alpha; KDR, kinase insert domain receptor; $\mathrm{GC}$, gastric cancer.

Furthermore, the ROC curve was plotted to evaluate the diagnostic value of PIG11 expression for patients with GC. The results suggested that PIG11 was able to distinguish GC tissues from adjacent tissues, indicating that PIG11 expression may act as a biomarker for GC. In addition, the protein expression of PIG11 in GC tissues was related to the T-, N-, Mand TNM stage, as well as CEA in blood serum. Furthermore, high levels of PIG11 protein expression were related to poor OS of patients with GC. Based on previous studies, the depth of tumor invasion and metastasis may affect patients in the early stages of tumor development, which has been considered to be the primary cause of death in cancer patients (50). Furthermore, the TNM stage has an important role in affecting the prognosis of patients with GC (51). Therefore, high expression of PIG11 may enhance the invasion and metastasis ability of GC cells and further lead to poor prognosis of patients with GC. PIG11 is expected to be an early biomarker for diagnosing GC. A bioinformatics analysis in the GEPIA database indicated that PIG11 expression is significantly correlated with GC-associated molecular markers, including TNF, CEACAM5, PIK3CA, VEGFA and KDR, which are related to the molecular typing and targeted drug therapy of GC, thereby indicating that highPIG11 expression maybe used as an independent molecular biomarker of GC.

Of note, the present study had certain limitations, which primarily include the following aspects: First, the present study was of a retrospective nature and all of the specimens were selected randomly, the size of the cohort was small and the quality of the specimens were limited, and the detection of the protein expression of PIG11 using IHC may have been affected by tumor heterogeneity and subjective scoring system analysis. Furthermore, the significant correlations among TNF, CEACAM5, PIK3CA, VEGFA, KDR and PIG11 based on the data from the GEPIA database require further detailed exploration for confirmation in a follow-up study due to the low R-values. The low R-values among TNF, CEACAM5, PIK3CA, VEGFA, KDR and PIG11 may be partly due to the low number of cases included in the GEPIA database. Finally, the biological roles of PIG11 in GC, particularly its role in apoptosis, remain to be elucidated. Future research should focus on the biological mechanisms at the cytological level and animal experiments to determine the role of PIG11 in GC.

In conclusion, the experimental results of the present study indicated that PIG11 is closely related to the invasion and metastasis of GC and prognosis of patients with GC, which was also confirmed by bioinformatics analysis. IG11 is expected to become a novel molecular marker for the clinical diagnosis and treatment of GC and a predictive indicator for the prognosis of GC patients.

\section{Acknowledgements}

Not applicable.

\section{Funding}

This study was supported by grants from the Nantong Science and Technology Program (grant no. MS12016031).

\section{Availability of data and materials}

The datasets used and/or analyzed during the current study are available from the corresponding author on reasonable request. 


\section{Authors' contributions}

JG performed data analyses and wrote the manuscript. SZ and XH contributed significantly to data analyses and manuscript revision. SC and YW conceived and designed the study. JG and XH performed the experiments. All authors read and approved the final manuscript.

\section{Ethics approval and consent to participate}

This study was approved by the Human Research Ethics Committee at the Affiliated Hospital of Nantong University (Nantong, China). All experimental methods and related protocols were performed according to the regulations of the Affiliated Hospital of Nantong University (Nantong, China). Written informed consent was obtained from the patients or their guardians.

\section{Patient consent for publication}

Not applicable.

\section{Competing interests}

The authors declare that they have no competing interests.

\section{References}

1. Li Y, Xu Q, Yang W, Wu T and Lu X: Oleanolic acid reduces aerobic glycolysis-associated proliferation by inhibiting yes-associated protein in gastric cancer cells. Gene 712: 143956, 2019.

2. Cavatorta O, Scida S, Miraglia C, Barchi A, Nouvenne A, Leandro G, Meschi T, De' Angelis GL and Di Mario F: Epidemiology of gastric cancer and risk factors. Acta Biomed 89: $82-87,2018$

3. Laterza L, Scaldaferri F and Gasbarrini A: Risk factors for gastric cancer: Is it time to discard PPIs? Gut 68: 176-177, 2019.

4. Venerito M, Vasapolli R, Rokkas T and Malfertheiner P: Gastric cancer: Epidemiology, prevention, and therapy. Helicobacter 23 (Suppl 1): e12518, 2018.

5. Rawla P and Barsouk A: Epidemiology of gastric cancer: Global trends, risk factors and prevention. Prz Gastroenterol 14: 26-38, 2019.

6. Zang YS, Dai C, Xu X, Cai X, Wang G, Wei J, Wu A, Sun W, Jiao $\mathrm{S}$ and $\mathrm{Xu}$ Q: Comprehensive analysis of potential immunotherapy genomic biomarkers in 1000 Chinese patients with cancer. Cancer Med 8: 4699-4708, 2019.

7. Arimoto T, Katagiri T, Oda K, Tsunoda T, Yasugi T, Osuga Y, Yoshikawa H, Nishii O, Yano T and Nakamura Y: Genome-wide cDNA microarray analysis of gene-expression profiles involved in ovarian endometriosis. Int J Oncol 22: 551-560, 2003.

8. Ricketts SL, Carter JC and Coleman WB: Identification of three $11 \mathrm{p} 11.2$ candidate liver tumor suppressors through analysis of known human genes. Mol Carcinog 36: 90-99, 2003.

9. Polyak K, Xia Y, Zweier J, Kinzler K and Vogelstein B: A model for p53-induced apoptosis. Nature 389: 300-305, 1997.

10. Liang XQ, Cao EH, Zhang Y and Qin JF: A P53 target gene, PIG11, contributes to chemosensitivity of cells to arsenic trioxide. FEBS Lett 569: 94-98, 2004.

11. Liang XQ, Cao EH, Zhang Y and Qin JF: P53-induced gene 11 (PIG11) involved in arsenic trioxide-induced apoptosis in human gastric cancer MGC-803 cells. Oncol Rep 10: 1265-1269, 2003.

12. Xu L, Hui A, Albanis E, Arthur M, O'Byrne S, Blaner W, Mukherjee P, Friedman S and Eng F: Human hepatic stellate cell lines, LX-1 and LX-2: New tools for analysis of hepatic fibrosis Gut 54: 142-151, 2005.

13. Liu XM, Xiong XF, Song Y, Tang RJ, Liang XQ and Cao EH: Possible roles of a tumor suppressor gene PIG11 in hepatocarcinogenesis and $\mathrm{As}_{2} \mathrm{O}_{3}$-induced apoptosis in liver cancer cells. J Gastroenterol 44: 460-469, 2009.
14. Wang Y, Liu X, Liu G, Wang X, Hu R and Liang X: PIG11 over-expression predicts good prognosis and induces HepG2 cell apoptosis via reactive oxygen species-dependent mitochondrial pathway. Biomed Pharmacother 108: 435-442, 2018.

15. Zhu B, Tian T and Zhao M: MiR-645 promotes proliferation and migration of non-small cell lung cancer cells by targeting TP53I11. Eur Rev Med Pharmacol Sci 24: 6150-6156, 2020.

16. Xiao T, Xu Z, Zhang H, Geng J, Qiao Y, Liang Y, Yu Y, Dong Q and Suo G: TP53I11 suppresses epithelial-mesenchymal transition and metastasis of breast cancer cells. BMB Rep 52: 379-384, 2019.

17. Li Z and Li Q: The latest 2010 WHO classification of tumors of digestive system. Zhonghua Bing Li Xue Za Zhi 40: 351-354, 2011 (In Chinese).

18. He X, Wu W, Lin Z, Ding Y, Si J and Sun L: Validation of the American Joint Committee on Cancer (AJCC) 8th edition stage system for gastric cancer patients: A population-based analysis. Gastric Cancer 21: 391-400, 2018.

19. Tang Z, Li C, Kang B, Gao G, Li C and Zhang Z: GEPIA: A web server for cancer and normal gene expression profiling and interactive analyses. Nucleic Acids Res 45: W98-W102, 2017.

20. Livak KJ and Schmittgen TD: Analysis of relative gene expression data using real-time quantitative PCR and the 2(-Delta Delta C(T)) method. Methods 25: 402-408, 2001.

21. Wang Y, Chen S, Tian W, Zhang Q, Jiang C, Qian L and Liu Y: High-expression $\mathrm{HBO} 1$ predicts poor prognosis in gastric cancer. Am J Clin Pathol 152: 517-526, 2019.

22. Camp R, Dolled-Filhart M and Rimm D: X-tile: A new bioinformatics tool for biomarker assessment and outcome-based cut-point optimization. Clin Cancer Res 10: 7252-7259, 2004.

23. Szasz AM, Lanczky A, Nagy A, Forster S, Hark K, Green JE, Boussioutas A, Busuttil R, Szabo A and Gyorffy B: Cross-validation of survival associated biomarkers in gastric cancer using transcriptomic data of 1,065 patients. Oncotarget 7: 49322-49333, 2016.

24. Röcken C: Molecular classification of gastric cancer. Expert Rev Mol Diagn 17: 293-301, 2017.

25. Cho JY, Lim JY, Cheong JH, Park YY, Yoon SL, Kim SM, Kim SB, Kim H, Hong SW, Park YN, et al: Gene expression signature-based prognostic risk score in gastric cancer. Clin Cancer Res 17: 1850-1857, 2011.

26. Ooi CH, Ivanova T, Wu J, Lee M, Tan IB, Tao J, Ward L, Koo JH, Gopalakrishnan V, Zhu Y, et al: Oncogenic pathway combinations predict clinical prognosis in gastric cancer. PLoS Genet 5: e1000676, 2009.

27. Guo S, Shang M, Dong Z, Zhang J, Wang Y and Zhao Y: The assessment of the optimal number of examined lymph nodes and prognostic models based on lymph nodes for predicting survival outcome in patients with stage N3b gastric cancer. Asia Pac J Clin Oncol: Aug 6, 2020 doi: 10.1111/ajco.13358 (Epub ahead of print).

28. Tan AC, Chan DL, Faisal W and Pavlakis N: New drug developments in metastatic gastric cancer. Therap Adv Gastroenterol 11: 1756284818808072,2018

29. Menard S, Pupa SM, Campiglio M and Tagliabue E: Biologic and therapeutic role of HER2 in cancer. Oncogene 22: 6570-6578, 2003.

30. Vasudev NS and Reynolds AR: Anti-angiogenic therapy for cancer: Current progress, unresolved questions and future directions. Angiogenesis 17: 471-494, 2014.

31. De Vita F, Borg C, Farina G, Geva R, Carton I, Cuku H, Wei R and Muro K: Ramucirumab and paclitaxel in patients with gastric cancer and prior trastuzumab: Subgroup analysis from RAINBOW study. Future Oncol 15: 2723-2731, 2019.

32. Troiani T, Napolitano S, Della Corte CM, Martini G, Martinelli E, Morgillo F and Ciardiello F: Therapeutic value of EGFR inhibition in CRC and NSCLC: 15 years of clinical evidence. ESMO Open 1: e000088, 2016.

33. Catenacci DVT, Tebbutt NC, Davidenko I, Murad AM, Al-Batran SE, Ilson DH, Tjulandin S, Gotovkin E, Karaszewska B, Bondarenko I, et al: Rilotumumab plus epirubicin, cisplatin, and capecitabine as first-line therapy in advanced MET-positive gastric or gastro-oesophageal junction cancer (RILOMET-1): A randomised, double-blind, placebo-controlled, phase 3 trial. Lancet Oncol 18: 1467-1482, 2017.

34. Shen X, Zhao Y, Chen X, Sun H, Liu M, Zhang W, Jiang F and Li P: Associations of PIK3CA mutations with clinical features and prognosis in gastric cancer. Future Oncol 15: 1873-1894, 2019. 
35. Xu DZ, Geng QR, Tian Y, Cai MY, Fang XJ, Zhan YQ, Zhou ZW, $\mathrm{Li}$ W, Chen YB, Sun XW, et al: Activated mammalian target of rapamycin is a potential therapeutic target in gastric cancer. BMC Cancer 10: 536, 2010.

36. Schoop I, Maleki S, Behrens H, Krüger S, Haag J and Röcken C: p53 immunostaining cannot be used to predict TP53 mutations in gastric cancer: Results from a large Central European cohort. Hum Pathol 105: 53-66, 2020.

37. Cancer Genome Atlas Research Network: Comprehensive molecular characterization of gastric adenocarcinoma. Nature 513: 202-209, 2014

38. Tannock I and Hickman J: Limits to personalized cancer medicine. N Engl J Med 375: 1289-1294, 2016.

39. Goussia AC, Papoudou-Bai A, Charchanti A, Kitsoulis P, Kanavaros P, Kalef-Ezra J, Stefanou D and Agnantis NJ: Alterations of p53 and Rb pathways are associated with high proliferation in bladder urothelial carcinomas. Anticancer Res 38: 3985-3988, 2018.

40. Ma Z, Yang Y, Di S, Feng X, Liu D, Jiang S, Hu W, Qin Z, $\mathrm{Li}$ Y, Lv J, et al: Pterostilbene exerts anticancer activity on non-small-cell lung cancer via activating endoplasmic reticulum stress. Sci Rep 7: 8091, 2017.

41. Navarro-Yepes J, Burns M, Anandhan A, Khalimonchuk O, del Razo LM, Quintanilla-Vega B, Pappa A, Panayiotidis MI and Franco R: Oxidative stress, redox signaling, and autophagy: Cell death versus survival. Antioxid Redox Signal 21: 66-85, 2014.

42. Yaprak E, Kasap M, Akpinar G, Kayaalti-Y üksek S, Sinanoğlu A, Guzel $\mathrm{N}$ and Demirturk Kocasarac H: The prominent proteins expressed in healthy gingiva: A pilot exploratory tissue proteomics study. Odontology 106: 19-28, 2018.

43. Kung CP, Khaku S, Jennis M, Zhou Y and Murphy ME: Identification of TRIML2, a novel p53 target, that enhances p53 SUMOylation and regulates the transactivation of proapoptotic genes. Mol Cancer Res 13: 250-262, 2015.
44. van Ginkel PR, Yan MB, Bhattacharya S, Polans AS and Kenealey JD: Natural products induce a $G$ protein-mediated calcium pathway activating p53 in cancer cells. Toxicol Appl Pharmacol 288: 453-462, 2015.

45. Lin CC, Lee IT, Wu WL, Lin WN and Yang CM: Adenosine triphosphate regulates NADPH oxidase activity leading to hydrogen peroxide production and COX-2/PGE2 expression in A549 cells. Am J Physiol Lung Cell Mol Physiol 303: L401-L412, 2012.

46. Chiarugi P: From anchorage dependent proliferation to survival: Lessons from redox signalling. IUBMB Life 60: 301-307, 2008.

47. Au S, Storey B, Moore J, Tang Q, Chen Y, Javaid S, Sarioglu A, Sullivan R, Madden M, O'Keefe R, et al: Clusters of circulating tumor cells traverse capillary-sized vessels. Proc Natl Acad Sci USA 113: 4947-4952, 2016.

48. Vlahakis A and Debnath $\mathrm{J}$ : The Interconnections between autophagy and integrin-mediated cell adhesion. J Mol Biol 429: 515-530, 2017.

49. Xiao T, Xu Z, Zhou Y, Zhang H, Geng J, Liang Y, Qiao H and Suo G: Loss of TP53I11 enhances the extracellular Matrix-independent survival by promoting activation of AMPK. IUBMB Life 71: 183-191, 2019.

50. Otterbein LR, Graceffa P and Dominguez R: The crystal structure of uncomplexed actin in the ADP state. Science 293: 708-711, 2001.

51. Harrison JD and Fielding JW: Prognostic factors for gastric cancer influencing clinical practice. World J Surg 19: 496-500, 1995. International (CC BY-NC-ND 4.0) License. 